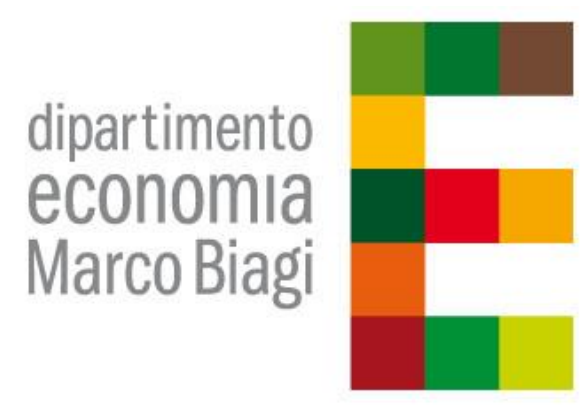

DEMB Working Paper Series

N. 105

Characterizing sets of lower bounds: a hidden convexity result

\author{
Emil Ernst*, Alberto Zaffaroni**
}

December 2016

* Aix Marseille University, CNRS

Address: Centrale Marseille, 12M, Marseille, France

email: emil.ernst@univ-amu.fr

** University of Modena and Reggio Emilia

Address: Viale Berengario 51, 41121 Modena, Italy

email: alberto.zaffaroni@unimore.it

ISSN: 2281-440X online

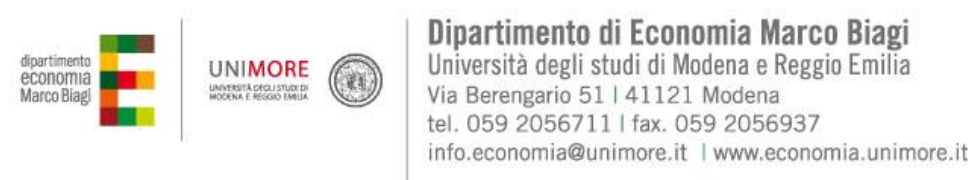




\title{
Characterizing sets of lower bounds: a hidden convexity result
}

\author{
Emil Ernst · Alberto Zaffaroni \\ Dedicated to Michel Théra in honor of his 70th birthday.
}

\begin{abstract}
This study addresses sets of lower bounds in a vector space ordered by a convex cone. It is easy to see that every set of lower bounds must be simultaneously downward and bounded from above, and must possesses the further property that it contains the supremum of any of its subsets which admits one. Our main result proves that these conditions are also sufficient, provided that the ordering cone is polyhedral. Simple counter-examples prove that the sufficiency fails when the polyhedrality assumption is dropped.
\end{abstract}

Keywords partially ordered vector spaces · polyhedral cone $\cdot$ set of lower bounds

Mathematics Subject Classification (2000) MSC 46A40 · MSC 06F20 · MSC 52B11

\section{Introduction}

Given $(X, \leq)$ a real vector space which is partially ordered by means of a pointed convex cone $K \subset X$, we shall call a non empty and proper subset $A$ of $X$ a set of lower bounds, if

$$
A=\{x \in X: x \leq y \quad \forall y \in S\}
$$

for some set $S \subset X$

When $(X, \leq)$ is a Dedekind complete vector lattice, that is when any subset of $X$ which is bounded from above possesses a supremum, it is easy to see that the only sets of lower bounds are the copies of the cone $-K$, that is the sets of the form $a-K$, with $a \in X$. To this respect, it is relevant to invoque the celebrated Choquet-Meyer theorem ([4]), saying that $(X, \leq)$ is a Dedekind complete vector lattice if and only if the ordering cone $K$ has a base which is a Choquet simplex, that is (see [3]) a convex set $C \subset X$ such that the intersection of any two homothetic copies of $\mathrm{C}$, if nonempty, is again a homothetic copy of $\mathrm{C}$, possibly degenerated into a point. When the dimension of $X$ is finite, additional information is provided by the Rogers-Shephard theorem (see [8]), which proves that a set is a Choquet

E. Ernst

Aix Marseille Univ, CNRS, Centrale Marseille, I2M, Marseille, France E-mail: emil.ernst@univ-amu.fr

A. Zaffaroni

Universitá di Modena e Reggio Emilia, Modena, Italy E-mail: alberto.zaffaroni@unimore.it 
simplex if and only if it is the convex hull of finitely many affinely independent points, as for instance when the vector space $X=\mathbb{R}^{n}$ is ordered by the cone $K=\mathbb{R}_{+}^{n}$.

In the general case of a partially ordered space $(X, \leq)$ which is not necessarily a Dedekind complete lattice, the class of sets of lower bounds lacks a clear description. Indeed, again as a consequence of the Choquet-Meyer theorem, it results that, every time when the ordering cone $K$ is non-simplicial, there are sets of lower bounds which are not mere copies of $-K$.

Let us notice that, regardless of specific assumption on the ordered vector space $(X, \leq)$, any set $A$ of lower bounds must satisfies each and every one of the following properties:

P1: $a_{1} \in A$ and $a_{2} \leq a_{1}$ imply $a_{2} \in A$ ( $A$ is downward);

P2: there exists $z \in X$ such that $a \leq z$, for all $a \in A$ ( $A$ is bounded from above);

P3: if $B \subseteq A$ admits a supremum $y=\sup B$, then $y \in A$ ( $A$ is sup-containing).

Indeed, any set of the form $a-K$, with $a \in X$, obviously satisfies all the above properties, and it is an easy task to prove that the intersection of a family of sets fulfills properties P1-3, provided that the same holds true for every set in the family. We will say that $A$ is regularly sup-containing if the three properties above are satisfied. It is then tempting to conclude that properties P1-3 completely characterize the sets of lower bounds, and thus state the following conjecture:

\section{$C: A$ non empty proper set $A \subset X$ is a set of lower bounds if and only if it is regularly sup-containing.}

Surprisingly enough, the validity of our conjecture strongly depends on the ordering cone. Thus, the main result of the present study (Theorem 1, Section 3), proves the conjecture for the particular case of a finite dimensional space $X$ ordered by a polyhedral cone $K$, while Proposition 7 (Section 4.3) describes the example of a pointed convex cone $K \subset \mathbb{R}^{2}$ for which the conjecture is false. Hence, the set of properties P1-3 either provides us with an accurate description of sets of lower bounds, or completely fails in doing so, depending on finely tuned properties of the ordering cone.

Let us also remark that a set of lower bounds is given by the intersection of all the copies of $-K$ containing it, being hence a convex set. To this respect, we may see Theorem 1 as being a hidden convexity result, as none of the properties P1-3 explicitly requires convexity, and since, as proved by Proposition 7, for a different choice of the ordering cone, some non-convex sets also satisfy properties P1-3.

Exploiting this convexity feature, we prove two different characterizations in dual terms. One is based on separation by means of the linear functionals which define the ordering cone. The other is based on the properties of the support function. These conditions are also specific for polyhedral orderings, as shown by a counterexample in $\mathbb{R}^{3}$.

Our main results, Theorem 1 and Proposition 7, lead us to state the open question of characterizing all the pointed convex cones $K$ such that the conjecture $C$ holds true. The concluding section of our study gives us the opportunity to discuss this question in some detail.

\section{Elementary properties of polyhedral cones in finite dimensional spaces}

We consider a finite dimensional partially ordered vector space $(X, \leq)$, in which the relation $\leq$ is induced by a convex cone $K$ which is pointed $(K \cap(-K)=\{0\})$ and solid (the interior 
of $K$ is nonempty). Given $\langle\cdot, \cdot\rangle$, some dot product on $X$, we denote by $K^{+}$its dual cone,

$$
K^{+}:=\{x \in X:\langle x, y\rangle \geq 0 \forall y \in K\}
$$

and by ext $K^{+}$the subset of $K^{+}$gathering all the extreme rays of $K^{+}$.

Given a subset $S \subseteq X$, we say that $z \in X$ is a lower (resp. upper) bound of $S$ if $z \leq s$ (resp. $s \leq z$ ) for all $s \in S$. Thus the set $L(S)$ of lower bounds of $S$ is given by

$$
L(S)=\{z \in X: z \leq s, \forall s \in S\}=\bigcap_{s \in S} s-K,
$$

while

$$
U(S)=\{z \in X: s \leq z, \forall s \in S\}=\bigcap_{s \in S} s+K
$$

stands for the set of upper bounds of $S$. The set $L(S)$ is thus defined as the intersection of all lower sections $l_{s}=L(\{s\})=\{z \in X: z \leq s\}$ with $s \in S$. If $L(S)$ admits a greatest element $y$, then $y$ is called the infimum of $S(y=\inf S)$; in this case $L(S)=l_{y}$. Analogously, if $U(S)$ admits a least element $v$, then $v$ is called supremum of $S$, indicated $v=\sup S$.

Despite its importance for the theory of ordered spaces, a good knowledge of the structure of sets of lower bound is not achieved (to our knowledge), and these notes are aimed to a deeper understanding of the properties of this kind of sets.

In this section, we gather several, more or less elementary, facts about pointed and solid polyhedral cones in a finite dimensional space $X$, that is pointed and solid cones which may be expressed as the intersection a finite number of closed half-spaces of $X$.

For each such cone $K$, there is a non empty and finite set $I$ of vectors from $X$ such that

$$
K=\{x \in X:\langle x, y\rangle \geq 0 \forall y \in I\} .
$$

Of course, for a given polyhedral cone $K$, the set $\mathcal{J}(K)$ of all the finite sets $I$ such that relation (1) holds true is infinite; let us denote by $F=\left\{f_{i}: i=\overline{1, m}\right\}$ an element of $\mathcal{J}(K)$ which is minimal with respect to set inclusion. Thus

$$
K=\left\{x \in X:\left\langle x, f_{i}\right\rangle \geq 0 \forall i=\overline{1, m}\right\} .
$$

The following obvious consequence of relation (2) needs no proof.

Lemma 1 Given two vectors $x, y \in X$, the two following facts are equivalent:

i) $x \leq y$

ii) $\left\langle x, f_{i}\right\rangle \leq\left\langle y, f_{i}\right\rangle \forall i=\overline{1, m}$.

As a consequence of the previous lemma, we are in a position to provide a first characterization of the set $L(S)$.

Proposition 1 Let $S$ be a nonempty and lower bounded set in $X$, and $\operatorname{set} \alpha_{k}=\inf _{s \in S}\left\langle s, f_{k}\right\rangle$, for $k=\overline{1, m}$. Then

$$
L(S)=\left\{z \in X:\left\langle z, f_{k}\right\rangle \leq \alpha_{k}, k=\overline{1, m}\right\} .
$$


Proof of Proposition 1: Let $x \in L(S)=\bigcap_{s \in S} s-K$. Then $x \in s-K$ for all $s \in S$, which yields $\left\langle s, f_{k}\right\rangle \geq\left\langle x, f_{k}\right\rangle$ for all $s \in S$ and all $k=\overline{1, m}$. Hence

$$
\left\langle x, f_{k}\right\rangle \leq \inf _{s \in S}\left\langle s, f_{k}\right\rangle=\alpha_{k}
$$

If conversely $\left\langle x, f_{k}\right\rangle \leq \alpha_{k}, k=\overline{1, m}$, and we take any $s \in S$, then we have $\left\langle s, f_{k}\right\rangle \geq \alpha_{k}$, and

$$
\left\langle s-x, f_{k}\right\rangle \geq 0 \quad \forall k=\overline{1, m}
$$

which, as a consequence of Lemma 1 , yields $s-x \in K$ for all $s \in S$, and therefore $x \in \bigcap_{s \in S} s-K$.

Since the set $F$ is minimal, it is possible to pick an element from $K$ such that all but one among the $m$ inequalities in (2) are strict, as indicated by the next result.

Lemma 2 For every $k=\overline{1, m}$, there exists $w_{k}$ in the boundary of $K$ such that

$$
\left\langle w_{k}, f_{k}\right\rangle=0, \quad\left\langle w_{k}, f_{i}\right\rangle>0 \quad \forall i=\overline{1, m}, i \neq k .
$$

Proof of Lemma 2: Let us pick $k=\overline{1, m}$; as $F$ is minimal in $\mathcal{J}(K)$ and $F \backslash\left\{f_{k}\right\} \subset F$, it follows that the closed convex cone

$$
K_{k}=\left\{x \in X:\left\langle x, f_{i}\right\rangle \geq 0 \forall i=\overline{1, m}, i \neq k\right\}
$$

is strictly larger than $K$. But $K$ is solid, so the larger cone $K_{k}$ must, at its turn, be solid. Moreover, like any convex set with non empty interior, $K_{k}$ is the closure of its interior. Hence the interior of $K_{k}$ cannot be completely contained in $K$, as this would imply that $K_{k}$ itself lies within $K$, a contradiction. One can thus find a vector, say $v_{k}$, laying in the interior of $K_{k}$ but outside $K$, that is

$$
\left\langle v_{k}, f_{k}\right\rangle<0, \quad\left\langle v_{k}, f_{i}\right\rangle>0 \quad \forall i=\overline{1, m}, i \neq k .
$$

Finally, let us pick $v$, a (non null) vector from the interior of $K$; hence

$$
\left\langle v, f_{i}\right\rangle>0 \quad \forall i=\overline{1, m}
$$

From relations (4) and (5) it results that there is some $\lambda_{k} \in(0,1)$ such that

$$
\left\langle w_{k}, f_{k}\right\rangle=0, \quad\left\langle w_{k}, f_{i}\right\rangle>0 \quad \forall i=\overline{1, m}, i \neq k
$$

where $w_{k}=\lambda_{k} v_{k}+\left(1-\lambda_{k}\right) v$. Obviously, such a vector $w_{k}$ must lie in the boundary of the cone $K$. 


\section{The main results}

3.1 Separating $L(S)$ and $S$ by means of linear functionals

Let $S$ be a nonempty set possessing at least a lower bound, and $f \in K^{+}$. It is obvious that

$$
\sup _{v \in L(S)}\langle v, f\rangle \leq \inf _{w \in S}\langle w, f\rangle ;
$$

this subsection addresses the study of the gap which may exists between the two sides of the above inequality.

Let us first consider the case of a set $S$ composed by two points $x, y \in X$ (the more general case of a finite set $S$ may be studied in a very similar way). It is a well known property (see [6] and also [1]) that, if $f \in \operatorname{ext} K^{+}$, then for every $\varepsilon>0$, there exists an element $z_{\varepsilon} \in X$ such that $z_{\varepsilon} \leq x$ and $z_{\varepsilon} \leq y$, and that

$$
\left\langle z_{\varepsilon}, f\right\rangle \geq \min (\langle x, f\rangle,\langle y, f\rangle)-\varepsilon
$$

in other words, the inequality (7) becomes an equality.

The following technical lemma exploits polyhedrality in order to extend to $\varepsilon=0$ relation (8).

Lemma 3 Given $x, y$, two vectors of $X$, and $k=\overline{1, m}$ a fixed index, there is an element $z_{k} \in X$ such that $z_{k} \leq x, z_{k} \leq y$, and

$$
\left\langle z_{k}, f_{k}\right\rangle=\min \left(\left\langle x, f_{k}\right\rangle,\left\langle y, f_{k}\right\rangle\right) .
$$

Proof of Lemma 3: Without restricting the generality, we may assume that $\left\langle x, f_{k}\right\rangle \leq$ $\left\langle y, f_{k}\right\rangle$, so

$$
\left\langle x, f_{k}\right\rangle=\min \left(\left\langle x, f_{k}\right\rangle,\left\langle y, f_{k}\right\rangle\right)
$$

Set

$$
s:=\frac{\left\langle y-x, f_{k}\right\rangle}{\left\langle v, f_{k}\right\rangle},
$$

where $v$ is a vector from the interior of $K$, and define

$$
y_{s}:=y-s v .
$$

Obviously, $s \geq 0$, so $y_{s} \leq y$, and

$$
\left\langle y_{s}, f_{k}\right\rangle=\left\langle y, f_{k}\right\rangle-s\left\langle v, f_{k}\right\rangle=\left\langle y, f_{k}\right\rangle-\frac{\left\langle y-x, f_{k}\right\rangle}{\left\langle v, f_{k}\right\rangle}\left\langle v, f_{k}\right\rangle=\left\langle x, f_{k}\right\rangle .
$$

Let us now define

$$
t_{i}:=\max \left(0, \frac{\left\langle y_{s}-x, f_{i}\right\rangle}{\left\langle w_{k}, f_{i}\right\rangle}\right), \quad \forall i=\overline{1, m}, i \neq k,
$$

where the vector $w_{k}$ is given by relation (3), and set

$$
t:=\max \left\{t_{i}: i=\overline{1, m}, i \neq k\right\} .
$$

Clearly, $t \geq 0$, so $y_{s}-t w_{k} \leq y_{s} \leq y$. Moreover,

$$
\left\langle y_{s}-t w_{k}, f_{k}\right\rangle=\left\langle y_{s}, f_{k}\right\rangle-t\left\langle w_{k}, f_{k}\right\rangle=\left\langle y_{s}, f_{k}\right\rangle,
$$


so from relation (10) it results that

$$
\left\langle y_{s}-t w_{k}, f_{k}\right\rangle=\left\langle x, f_{k}\right\rangle
$$

while

$$
\begin{aligned}
\left\langle y_{s}-t w_{k}, f_{i}\right\rangle & =\left\langle y_{s}, f_{i}\right\rangle-t\left\langle w_{k}, f_{i}\right\rangle \\
& \leq\left\langle y_{s}, f_{i}\right\rangle-\frac{\left\langle y_{s}-x, f_{i}\right\rangle}{\left\langle w_{k}, f_{i}\right\rangle}\left\langle w_{k}, f_{i}\right\rangle=\left\langle x, f_{i}\right\rangle \quad \forall i=\overline{1, m}, i \neq k .
\end{aligned}
$$

Relations (11), (12) and Lemma 1 prove that $y_{s}-t w_{k} \leq x$. Accordingly, the vector $y_{s}-t w_{k}$ is less then both $x$ and $y$, and (in virtue of relation (11)) it also satisfies relation (9); Lemma 3 is thus fulfilled for $z_{k}:=y_{s}-t w_{k}$.

The case of a non necessarily finite set $S$ is much more complicated. Indeed, standard examples (see for instance the one depicted in subsection 4.2) prove that the gap between $\sup _{v \in L(S)}\langle v, f\rangle$ and $\inf _{w \in S}\langle w, f\rangle$ may be non null even for vectors $f$ lying in ext $K^{+}$.

When $K$ is polyhedral, we can prove that the inequality in (7) becomes an equality. Thus, for any given $f_{k} \in F$, we can deduce the largest value of $f_{k}$ on $L(S)$ only by looking at the values of $f_{k}$ on $S$.

Proposition 2 Given a nonempty and bounded from below set $S \subseteq X$, and $f \in F$, then it holds that

$$
\sup _{v \in L(S)}\langle v, f\rangle=\inf _{w \in S}\langle w, f\rangle
$$

Proof of Proposition 2: Reasoning by contradiction, assume that, for some $k=\overline{1, m}$, it holds that

$$
\alpha_{k}:=\inf _{w \in S}\left\langle w, f_{k}\right\rangle>\sup _{v \in L(S)}\left\langle v, f_{k}\right\rangle=: \beta_{k},
$$

and choose $y \in X$ such that $\left\langle y, f_{k}\right\rangle=\gamma \in\left(\beta_{k}, \alpha_{k}\right)$.

Now take $w_{k}$ as in Lemma 2 and define

$$
t_{i}=\frac{\left\langle y, f_{i}\right\rangle-\alpha_{i}}{\left\langle w_{k}, f_{i}\right\rangle}, \quad \forall i=\overline{1, m}, i \neq k,
$$

$t=\max _{i \neq k} t_{i}$, and $y_{k}=y-t w_{k}$. Then we have, for all $i \neq k$,

$$
\left\langle y_{k}, f_{i}\right\rangle=\left\langle y, f_{i}\right\rangle-t\left\langle w_{k}, f_{i}\right\rangle \leq\left\langle y, f_{i}\right\rangle-t_{i}\left\langle w_{k}, f_{i}\right\rangle=\alpha_{i} .
$$

Moreover we have

$$
\left\langle y_{k}, f_{k}\right\rangle=\left\langle y, f_{k}\right\rangle-t\left\langle w_{k}, f_{k}\right\rangle=\left\langle y, f_{k}\right\rangle=\gamma<\alpha_{k} .
$$

Thus it holds

while the inequality

$$
\left\langle y_{k}, f_{i}\right\rangle \leq \alpha_{i}, \quad \forall i=\overline{1, m},
$$

$$
\left\langle y_{k}, f_{k}\right\rangle=\gamma>\beta_{k}=\sup _{z \in L(S)}\left\langle z, f_{k}\right\rangle
$$

shows that $y_{k} \notin L(S)$, contradicting in this way Proposition 1 .

By a geometric point of view, we can visualize the previous result by saying that $S$ and $A=L(S)$ are separated with no gap by every functional in $F$, provided that the ordering cone $K$ is polyhedral. 


\subsection{Direct and dual characterizations of a set of lower bounds}

The results presented above become relevant in the description of sets of lower bounds in the general setting in which the vectors $f_{1}, \ldots, f_{m}$ are not linearly independent, and thus Choquet-Meyer's Theorem does not apply.

Let us start by making more precise the description of a set of lower bounds as intersection of lower sections. A lower section $l_{x}$ is a translation of the ordering cone, $l_{x}=x-K$, and a set of lower bounds is the intersection of copies of $-K$, so that $A$ is a set of lower bounds if and only if it satisfies the following separation property:

$$
\forall y \notin A, \exists z \in X: A \subseteq z-K \text { and } y \notin z-K .
$$

Using relation (14), we obtain a description of sets $A$ of lower bounds by means of copies of $-K:$ a description "from outside". This description is somehow analogous to the well-known characterization "from outside" of closed convex sets as intersection of closed half-spaces. Of course, the family of closed, convex sets can also be described "from inside"; conjecture $C$ attempts to provide an analogous "from inside" description for the case of sets of lower bounds.

Despite being a simple rewording of the definition, this separation property is worth being emphasized. While there are many instances in which separation is used to characterize classes of sets more general than convex ones, here the elementary separating sets are convex sets of a special type, and thus we obtain a kind of separation which is less general than the one in which half-spaces are used, and we can use relation (14) to characterize a particular subclass of convex sets in $X$.

A very similar situation is encountered for instance when closed balls of some normed space $X$ are used as separating sets in the "from outside" description: in this framework, it can be seen (see [5]) that every Banch space whose norm is Frechet differentiable enjoys the so-called Mazur Intersection Property, that is every closed, bounded, convex set is the intersection of closed balls containing it.

The following result offers the first characterization of sets of lower bounds. It can be seen as a dual description, in terms of extreme functionals in $F$, of the separation property (14) by means of copies of $-K$, which holds for sets of lower bounds.

Proposition 3 The set $A \subset X$ is a set of lower bounds if and only if it is upper bounded and for every $y \notin A$ there exists $f \in F$ such that

$$
\langle y, f\rangle>\sup _{a \in A}\langle a, f\rangle .
$$

Proof of Proposition 3: If $A=L(S)$ for some set $S \subset X$ and $y \notin A$, then there exists $s \in S$ such that $y \notin s-K$ and $A \subseteq s-K$. The latter implies $\sup _{a \in A}\left\langle a, f_{i}\right\rangle \leq\left\langle s, f_{i}\right\rangle$ for all $i=\overline{1, m}$. Moreover there exists $k=\overline{1, m}$ such that $\left\langle y, f_{k}\right\rangle>\sup _{x \in s-K}\left\langle x, f_{k}\right\rangle$. Observe that $\sup _{x \in s-K}\left\langle x, f_{i}\right\rangle=\left\langle s, f_{i}\right\rangle$, for all $i=\overline{1, m}$, so that $\left\langle y, f_{k}\right\rangle>\sup _{a \in A}\left\langle a, f_{k}\right\rangle$.

To prove the converse we will show that (14) holds. To this aim, consider $y \notin A$ and apply the assumptions to find $f \in F$ such that $\langle y, f\rangle>\sup _{a \in A}\langle a, f\rangle$. Now use Proposition 2 with $S=U(A)$, so that $\langle y, f\rangle>\inf _{s \in S}\langle s, f\rangle$ and there exists $z \in U(A)$ such that $\langle y, f\rangle>\langle z, f\rangle=\sup _{x \in z-K}\langle x, f\rangle$, and $y \notin z-K$. Moreover $z \in U(A)$ means that $A \subseteq z-K$, and the result is proved. 
Remark 1 The type of linear separation indicated in Proposition 3 was named $H$-convexity by Boltyanski [2]. Given a normed space $X$ and a family $H \subseteq S^{*}$, the dual unit sphere, it refers to the possibility of representing a set $A$ as the intersection of closed halfspaces defined by means of functionals in $H$. The original definition also asks that $H$ is not onesided, that is $H$ is not included in a closed halfsphere of $S^{*}$. This does not hold true for $F$. But for instance [7] drops the latter requirement.

As a consequence of Proposition 2, it is possible to provide a description of the sets $B \subset X$ possessing a supremum $y=\sup B$.

Proposition 4 Given $B \subset X$, then it holds that $y=\sup B$ if and only if

$$
\sup _{b \in B}\left\langle b, f_{i}\right\rangle=\left\langle y, f_{i}\right\rangle, \quad \forall i=\overline{1, m}
$$

Proof of Proposition 4: If relation (15) holds, then we have

$$
\left\langle b-y, f_{i}\right\rangle \leq 0, \quad \forall b \in B, \quad \forall i=\overline{1, m}
$$

which yields $B \subset y-K$. In order to show that $B=y-K$, suppose by contradiction that there exists $w \in U(B)$ such that $w-y \notin K$. Then there exists $k=\overline{1, m}$ such that $\left\langle w-y, f_{k}\right\rangle<0$.

Since $B \subset w-K$, then

$$
\sup _{b \in B}\left\langle b, f_{k}\right\rangle \leq \sup _{z \in w-K}\left\langle z, f_{k}\right\rangle=\left\langle w, f_{k}\right\rangle<\left\langle y, f_{k}\right\rangle
$$

But the strict inequality in (16) contradicts (15).

Conversely, let us consider $B$ a set admitting a supremum, and set $y=\sup B$. Clearly, $U(B)=y+K$ and relation

$$
\sup _{b \in B}\left\langle b, f_{i}\right\rangle=\inf _{z \in y+K}\left\langle z, f_{i}\right\rangle=\left\langle y, f_{i}\right\rangle, \quad \forall i=\overline{1, m} .
$$

stems from Proposition 2.

We are now in a position to prove the conjecture $C$ for the particular case of a finite dimensional vector space ordered by a cone which is pointed, solid and polyhedral.

Our results says that a $A \subset X$ is a set of lower bounds if and only if it is regularly supcontaining. Thus, we obtain the desired "from inside" description of sets of lower bounds. Moreover, we prove that regularly sup-containing sets are automatically closed and convex, provided that the ordering cone is polyhedral.

Theorem 1 Given a nonempty proper subset $A \subset X$ satisfying relations $P 1-3$, let us set $\alpha_{i}:=\sup _{A}\left\langle\cdot, f_{i}\right\rangle$, and define $S:=U(A)=\left\{x \in X:\left\langle x, f_{i}\right\rangle \geq \alpha_{i}, \quad \forall i=\overline{1, m}\right\}$. Then

$$
A=\left\{x \in X:\left\langle x, f_{i}\right\rangle \leq \alpha_{i}, \quad \forall i=\overline{1, m}\right\}=L(S) .
$$


Proof of Theorem 1: We deduce from Proposition 1 that $L:=\left\{x \in X:\left\langle x, f_{i}\right\rangle \leq\right.$ $\left.\alpha_{i}, \quad \forall i=\overline{1, m}\right\}$ is the set of lower bounds of $S$. Then we need to show that $A=L$. As the relation $A \subseteq L$ is obvious, we have to prove that

$$
A \supset\left\{x \in X:\left\langle x, f_{i}\right\rangle \leq \alpha_{i}, \forall i=\overline{1, m}\right\} .
$$

To this aim, let us consider an element $x \in X$ such that

$$
\left\langle x, f_{i}\right\rangle \leq \alpha_{i}, \quad \forall i=\overline{1, m},
$$

and let us pick $k=\overline{1, m}$. Since $\alpha_{k}$ is the supremum of the function $\left\langle\cdot, f_{k}\right\rangle$ over $A$, it results that there is a sequence $\left(y_{k, n}\right)_{n \in \mathbb{N}} \in A$ such that

$$
\lim _{n \rightarrow+\infty}\left\langle y_{k, n}, f_{k}\right\rangle=\alpha_{k}
$$

By applying the conclusion of Lemma 3 to $x$ and $y_{k, n}$, we deduce that there exists a vector $z_{k, n}$ such that $z_{k, n} \leq y_{k, n}, z_{k, n} \leq x$, and

$$
\left\langle z_{k, n}, f_{k}\right\rangle=\min \left(\left\langle x, f_{k}\right\rangle,\left\langle y_{k, n}, f_{k}\right\rangle\right) .
$$

We have thus defined a sequence $\left(z_{k, n}\right)_{n}$ of elements from $A$ ( $z_{k, n}$ belongs to $A$ since $A$ is a downward set, $y_{k, n} \in A$ and $z_{k, n} \leq y_{k, n}$ ), which is also bounded above by $x$ (indeed, $\left.z_{k, n} \leq x\right)$. If moreover we let $n$ go to $+\infty$ in (19), we deduce that

$$
\lim _{n \rightarrow+\infty}\left\langle z_{k, n}, f_{k}\right\rangle=\min \left(\lim _{n \rightarrow+\infty}\left\langle y_{k, n}, f_{k}\right\rangle,\left\langle x, f_{k}\right\rangle\right)=\min \left(\alpha_{k},\left\langle x, f_{k}\right\rangle\right)=\left\langle x, f_{k}\right\rangle .
$$

Accordingly, the set

$$
B:=\left\{z_{i, n}: i=\overline{1, m}, n \in \mathbb{N}\right\}
$$

is a subset of $A$, it is bounded from above by $x$, and, by virtue of relation (20), it holds that

$$
\sup _{B}\left\langle\cdot, f_{i}\right\rangle=\left\langle x, f_{i}\right\rangle \quad \forall i=\overline{1, m} .
$$

Obviously, $x$ is an upper bound for $B$, and, if $w \in X$ is another upper bound for $B$, it follows from Lemma 1 that

$$
\left\langle w, f_{i}\right\rangle \geq \sup _{B}\left\langle\cdot, f_{i}\right\rangle \quad \forall i=\overline{1, m}
$$

combine relations (21), (22) and Lemma 1 to conclude that $x$ is lower than any upper bound of $B$, that is that $x$ is the supremum of the subset $B$ of $A$.

Since $A$ satisfies property $\mathrm{P} 3$, it yields that $x$ is an element of $A$, so relation (18) is true and the proof of Theorem 1 is completed.

Once we are able to identify the sets of lower bounds as the ones which are regularly sup-containing, then we can exploit properties P1-3 to obtain a dual characterization of a set $A$ of lower bounds in terms of its support function $\sigma_{A}: X^{*} \rightarrow \mathbb{R}_{\infty}=\mathbb{R} \cup+\infty$, given by

$$
\sigma_{A}(f)=\sup _{a \in A}\langle a, f\rangle .
$$

Indeed it is easy to see that, by $\mathrm{P} 1-2$, the effective domain of $\sigma_{A}$ (also called the barrier cone of $A$ ) is given by $K^{+}$. Moreover, property $P 3$ implies that $\sigma_{A}$ is as flat as possible. To be more clear, in the particular case where $A=z-K$ for some $z \in X$, then $\sigma_{A}(f)=\langle z, f\rangle$ for all $f \in K^{+}$, hence $\sigma_{A}$ is linear on $K^{+}$. In greater generality, we can prove that, provided $\sigma_{A}$ majorizes a linear function on $F$, then it majorizes the same function on $K^{+}$. 
Proposition 5 The set $A \subseteq X$ is a set of lower bounds if and only if its support function $\sigma_{A}$ satisfies:

i) the effective domain of $\sigma_{A}$ is $K^{+}$;

ii) if $\langle y, f\rangle \leq \sigma_{A}(f), \quad \forall f \in F, \quad$ then $\quad\langle y, \ell\rangle \leq \sigma_{A}(\ell), \quad \forall \ell \in K^{+}$.

Proof of Proposition 5: Property P1 yields $\sigma_{A}(\ell) \leq \sigma_{z-K}(\ell)=\langle z, \ell\rangle$, for all $\ell \in K^{+}$, while P2 says that $\sigma_{A}(\ell)=+\infty$ if $\ell \notin K^{+}$, so that the validity of $\mathrm{P} 1$ and $\mathrm{P} 2$ is equivalent to $(i)$. Moreover the inclusion $B \subseteq A$ is equivalent to $\sigma_{B} \leq \sigma_{A}$ on $X^{*}$, while the relation $y=\sup B$ amounts to $\sigma_{B}(f)=\langle y, f\rangle$ for all $f \in F$. Hence P3 becomes $(i i)$.

\section{Three examples}

In this section we show by means of three examples that our assumptions cannot be completely dispensed with.

\subsection{Partially ordered sets}

The notions of lower and upper bounds, infimum and supremum, and (regularly) sup-containing set, can be easily extended to partially ordered sets which are not linear spaces. The following example shows that conjecture $\mathrm{C}$ does not hold in this setting, that is a regular sup-containing set $A$ is not necessarily a set of lower bounds, even if the ordering relation is given component-wise, hence deduced by a simplicial cone.

Let indeed $X=\{a, b, c, d, e\} \subseteq \mathbb{R}^{2}$ with $a=(2,2), b=(1,1), c=(0,3), d=(5,4)$ and $e=(4,5)$ with the component-wise order relation $\leq$, and let $A=\{b, c\}$. It is easy to see that $A$ is regularly sup-containing but it is not a set of lower bounds.

\subsection{An example in $\mathbb{R}^{3}$}

The assumption that the ordering cone is polyhedral in Proposition 2 cannot be extended to include any closed, convex, pointed, solid cone $K$. Indeed consider $X=\mathbb{R}^{3}$ with $K=$ $\left\{(x, y, z) \in \mathbb{R}^{3}: z \geq \sqrt{x^{2}+y^{2}}\right\}$ the so-called Lorentz cone. Let $A=-K \cap H$, with $H=$ $\{(x, y, z): x+z \leq-1\}$. The set $A$ is not a set of lower bounds, as it holds $K=U(A)$, so that $A$ admits the origin as its supremum, and the intersection of all copies of $-K$ containing $A$ is $-K$, which is different from $A$. For all linear functionals $f$ which are extreme directions of $K^{+}=K$, it holds $\sup _{a \in A}\langle a, f\rangle=0$, except for (the ones proportional to) $\bar{f}=(1,0,1)$, for which $\sup _{a \in A}\langle a, \bar{f}\rangle=-1$ holds.

This example contradicts Proposition 2 as the valutation of $\bar{f}$ on $A$ and $U(A)$ shows a gap; and one of the implications in both Propositions 3 and 4.

\subsection{An example in $\mathbb{R}^{2}$}

As for the validity of Theorem 1 , we address the case when $X=\mathbb{R}^{2}$ and $K=\{(0,0)\} \cup$ $\mathbb{R}_{++}^{2}$. In this particular setting, the following very strong result is in order.

Proposition 6 If the supremum of a set exists, then it belongs to the set itself. 
Proof of Proposition 6: Let $x=\left(x_{1}, x_{2}\right) \in \mathbb{R}^{2}$ be the supremum of the set $A \subset \mathbb{R}^{2}$. Since $x$ is the smallest upper bound for $A$, and since $x$ and $y:=\left(x_{1}+1, x_{2}\right)$ are incomparable (indeed, the vector $y-x=(1,0)$ belongs neither to $K$, nor to $-K$ ), we can conclude that $y$ is not an upper bound of $A$.

Consequently, $A$ does not entirely lie in $y-K$; as on the other hand, $A$ is contained in $x-K$, it follows that $A$ has at least an element in $(x-K) \backslash(y-K)$ :

$$
A \cap((x-K) \backslash(y-K)) \neq \emptyset .
$$

Or, $(x-K) \backslash(y-K)=\{x\}$, so $A \cap\{x\} \neq \emptyset$, which means that $x$ belongs to $A$.

In this case, any set satisfies property $\mathrm{P} 3$, and the following result is not a surprise.

Proposition 7 The set

$$
A=((-1,0)-K) \cup((0,-1)-K)
$$

fulfills properties P1-3, yet it is not a set of lower bounds.

Proof of Proposition 7: It is obvious that any union of copies of $-K$ satisfies property $\mathrm{P} 1$; in particular, the set $A$, as being the union of two copies of $-K$, fulfills this requirement.

It is again easy to see that $(1,1)$ is an upper bound for $A$, so property $\mathrm{P} 2$ is equally fulfilled.

Since any subset of $\mathbb{R}^{2}$ satisfies property $\mathrm{P} 3$, it means that we have proved that all conditions P1-3 are fulfilled by the set $A$.

On the other hand, although the vectors $(-1,0)$ and $(0,-1)$ are members of $A$, their mid-point, $\left(-\frac{1}{2},-\frac{1}{2}\right)$ does not belongs to $A$ (indeed, $A$ can alternatively be described as the union of all the vectors lower than at least one between the two vectors $(-1,0)$ and $(0,-1)$; and $\left(-\frac{1}{2},-\frac{1}{2}\right)$ is incomparable to both the vectors $(-1,0)$ and $\left.(0,-1)\right)$.

Accordingly, the set $A$ is not convex, so it cannot be a set of lower bounds.

\section{Conclusions}

In the case of a finite dimensional vector space ordered by a cone which, in addition to being pointed and solid, is also polyhedral, Theorem 1 provides us with a simple manner to verify if a given set is a set of lower bounds. Indeed, in order to decide if a set $A$ is the intersection of a family of copies of the cone $-K$, it suffices to verify that $A$ is a bounded from above downward set, which also contains the supremum of each of its subsets possessing one.

When the polyhedrality of the ordering cone is dropped, Proposition 7 educates us about the possibility that a non-convex set verifies conditions P1-3. It is hence natural to ask under what assumptions on the ordering cone $K$, fulfilling conditions P1-3 is sufficient for a set to be a set of lower bounds. At our best knowledge, this question is open, and no simple solution seems in reach.

An important step in answering this question is to completely understand the role of the polyhedrality of the ordering cone in the validity of the conjecture $C:$ an exemple of a non-polyhedral cone granting that the conjecture $C$ holds true will be pivotal for a detailed analysis of this problem. 


\section{References}

1. Benoist J., Borwein J.M., Popovici N, A characterization of quasiconvex vector-valued functions, Proc. Amer. Math. Soc., 131, pp. 1109-1113 ( 2003)

2. Boltyanski, V., On certain classes of convex sets, Soviet Math. Doklady, 17, p. 10-13 (1976)

3. Choquet, G, Unicité des représentations intégrales au moyen de points extrémaux dans les cônes convexes réticulés, C. R. Acad. Sci. Paris, 243, p. 555-557 (1956)

4. Choquet, G., Meyer, P.A. Existence et unicité des représentations intégrales dans les ensembles convexes compacts quelconques, Ann. Inst. Fourier, 13, p. 139-154 (1963)

5. Granero, A.S., Jimenez-Sevilla, M., Moreno, J.P., Intersections of Closed Balls and Geometry of Banach Spaces, Extracta mathematicae, 19, p. 55-92 (1992)

6. Jameson G., Ordered Linear Spaces, Lecture Notes in Mathematics 141, Springer Verlag, (1970)

7. Moreno, J.P., Schneider, R., Continuity properties of the ball hull properties, Nonlinear Analysis, 66, $p$ 914-925 (2007)

8. Rogers, C.A., Shepard G.C., The difference body of a convex body, Arch. Math., 8, p. 220-233 (1957) 